\title{
Factores de riesgo para desarrollar diabetes mellitus II
}

\author{
Risk factors for developing diabetes mellitus II \\ Factores de risco para o desenvolvimento da diabetes mellitus II
}

\author{
Gabriela Margoth Uyaguari-Matute ${ }^{1}$ \\ gabmargothum_12@hotmail.com \\ ORCID: 0000-0002-5340-3626
}

Andrés Alexis Ramírez-Coronel1,2,3

andres.ramírez@ucacue.edu.ec

ORCID: 0000-0002-6996-0443

\author{
Isabel Cristina Mesa-Cano1,2 \\ imesac@ucacue.edu.ec \\ ORCID: 0000-0003-3263-6145
}

Pedro Carlos Martínez-Suárez
pmartinezs@ucacue.edu.ec
ORCID: 0000-0002-1441-3821

\author{
${ }^{1}$ Maestría en Gestión del Cuidado del Posgrados de la Universidad Católica de Cuenca, Ecuador \\ ${ }^{2}$ Carrera de Enfermería de la Universidad Católica de Cuenca, Ecuador \\ ${ }^{3}$ Laboratorio de Psicometría, Psicología Comparada y Etología del Centro de Investigación, Innovación y \\ Transferencia de Tecnología (CIITT) de la Universidad Católica de Cuenca, Ecuador \\ ${ }^{4}$ Facultad de Psicología Clínica de la Universidad Católica de Cuenca, Ecuador
}

Recibido 20 de noviembre 2020 | Arbitrado y aceptado 9 de diciembre 2020 | Publicado en 04 de enero 2021

\begin{abstract}
RESUMEN
La Diabetes mellitus constituye uno de los problemas de salud más importantes en el mundo por la alta carga de enfermedad en términos de discapacidad y mortalidad prematura que ocasiona, debido a su alta prevalencia e incidencia a nivel mundial se configura como una epidemia. Objetivo. Determinar el riesgo para desarrollar diabetes mellitus tipo II en las personas de la Cuidad de Cuenca. Materiales y métodos. Se realizó un estudio, descriptivo, correlacional, prospectivo, cuantitativo y de corte transversal, mediante la aplicación del test de FINDRISC, La muestra estuvo compuesta por 379 personas no diabéticas con edades comprendidas entre 18 y 65 años de edad. Resultados. Se determinó que las variables sexo, edad, el peso, la talla, el perímetro de cintura, el tiempo diario de actividad física y antecedentes familiares de DM1- 2 presentan una correlación significativa en el nivel 0,01 (bilateral) con el riesgo de desarrollar DM2 según la puntuación del test de FINDRISC. Conclusión. Los datos obtenidos sugieren la necesidad de programas de intervención multidisciplinar en las unidades de salud asociados a programas educativos, ingesta dietética adecuada y actividad física regular.
\end{abstract}

Palabras clave: Cuestionario FINDRISC; factores relacionados; Diabetes mellitus 2

\begin{abstract}
Diabetes mellitus is one of the most important health problems in the world due to the high burden of disease in terms of disability and premature mortality it causes, due to its high prevalence and incidence worldwide is configured as an epidemic. Objective. To determine the risk of developing type II diabetes mellitus in people from the city of Cuenca. Materials and methods. Descriptive, correlational, prospective, quantitative and cross-sectional study was carried out through the application of the FINDRISC test. The sample was composed of 379 non-diabetic persons between 18 and 65 years of age. Results. It was determined that the variables sex, age, weight, height, waist circumference, daily physical activity time and family history of DM1- 2 presented a significant correlation at the 0.01 level (bilateral) with the risk of developing DM2 according to the FINDRISC test score. Conclusion. The data obtained suggest the need for multidisciplinary intervention programs in health units associated with educational programs, adequate dietary intake and regular physical activity.
\end{abstract}

Key words: FINDRISC test; relationship factors; Diabetes mellitus 2

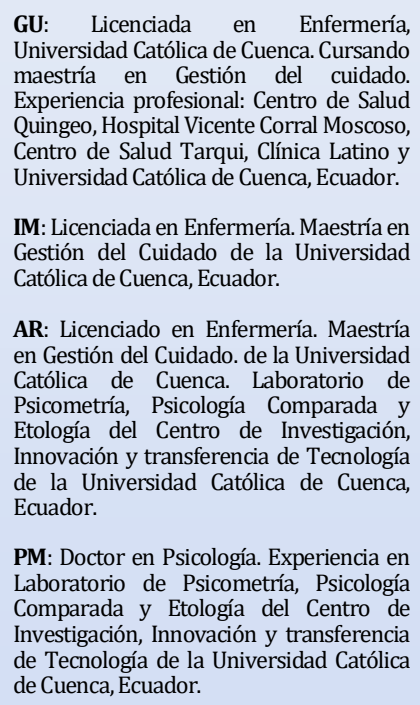

GU: Licenciada en Enfermería, Universidad Católica de Cuenca. Cursando maestría en Gestión del cuidado. Experiencia profesional: Centro de Salud Quingeo, Hospital Vicente Corral Moscoso, Centro de Salud Tarqui, Clínica Latino y Universidad Católica de Cuenca, Ecuador

IM: Licenciada en Enfermería. Maestría en Gestión del Cuidado de la Universidad Católica de Cuenca, Ecuador.

AR: Licenciado en Enfermería. Maestría en Gestión del Cuidado. de la Universidad Católica de Cuenca. Laboratorio de Psicometría, Psicología Comparada y Etología del Centro de Investigación, Etología del Centro de Investigación, de la Universidad Católica de Cuenca, Ecuador.

PM: Doctor en Psicología. Experiencia en Laboratorio de Psicometría, Psicología Comparada y Etoloǵa del Centro de Investigación Innovación y transfero de de Tecnología de la Universidad Católica de Tecnología de la
de Cuenca, Ecuador. 
GU: Licenciada en Enfermería, Universidad Católica de Cuenca. Cursando maestría en Gestión del cuidado. Experiencia profesional: Centro de Salud Quingeo, Hospital Vicente Corral Moscoso, Centro de Salud Tarqui, Clínica Latino y Universidad Católica de Cuenca, Ecuador.

IM: Licenciada en Enfermería. Maestría en Gestión del Cuidado de la Universidad Católica de Cuenca, Ecuador.

AR: Licenciado en Enfermería. Maestría en Gestión del Cuidado. de la Universidad Católica de Cuenca. Laboratorio de Psicometría, Psicología Comparada y Etología del Centro de Investigación, Etología del Centro de Investigación, Innovación y trans de la Universidad Católica de Cuenca, Ecuador.

PM: Doctor en Psicología. Experiencia en Laboratorio de Psicometría, Psicología Comparada y Etología del Centro de Investigación, Innovación y transferencia de Tecnoló, de la Universidad Católica de Cuenca, Ecuador.

\begin{abstract}
RESUMO
A diabetes mellitus é um dos problemas de saúde mais importantes do mundo devido ao elevado peso da doença em termos de incapacidade e mortalidade prematura que provoca, devido à sua elevada prevalência e incidência a nível mundial é configurada como uma epidemia. Objectivo. Determinar o risco de desenvolvimento da diabetes mellitus tipo II nas pessoas da cidade de Cuenca. Materiais e métodos. Foi realizado um estudo descritivo, correlativo, prospectivo, quantitativo e transversal utilizando o teste FINDRISC. A amostra consistiu em 379 pessoas não diabéticas entre os 18 e os 65 anos de idade. Resultados. Foi determinado que as variáveis sexo, idade, peso, altura, circunferência da cintura, tempo de atividade física diária e história familiar de DM1- 2 apresentaram uma correlação significativa ao nível 0,01 (bilateral) com o risco de desenvolvimento de DM2 de acordo com a pontuação do teste FINDRISC. Conclusão. Os dados obtidos sugerem a necessidade de programas de intervenção multidisciplinar em unidades de saúde associados a programas educativos, ingestão alimentar adequada e actividade física regular.
\end{abstract}

Palavras-chave: Questionário FINDRISC, factores relacionados, Diabetes mellitus 2

\section{INTRODUCCIÓN}

$\mathrm{L}$ a diabetes es una de las enfermedades no transmisibles más comunes a escala mundial se considera como una epidemia en muchos países desarrollados y recientemente industrializados, especialmente la diabetes tipo 2, que se ha constituido en un enorme problema de salud pública y de costos altos, ocupando los diez primeros lugares como consulta médica y hospitalización a nivel mundial (1).

En Europa y EE.UU. la diabetes mellitus constituye la mayor causa de morbimortalidad y de gasto sanitario con una incidencia que progresa de manera alarmante. En Europa se calcula que el número de personas con diabetes en 2011 fue de 52,6 millones lo que representa el $8,1 \%$ de la población adulta, siendo Rusia el país con mayor prevalencia e incidencia. la prevalencia de la diabetes mellitus 2 se incrementa con la edad por lo que en Europa se estima que para el año 2030 la población mayor de 50 años será más del $40 \%$ de la población con las complicaciones que esta trae (2).
Cabe mencionar que, según el último reporte de la IDF (International Diabetes Federation) 2015, China es el país con mayor número de pacientes diabéticos adultos con un total de 109.6 millones de personas (2).

La Federación Internacional de Diabetes (FID) calcula actualmente que los adultos de 20 a 79 años el $9.3 \%$ tienen diabetes, una prevalencia de 463 millones de personas. En los adolescentes menores de 20 años y los niños se calcula que los 1,1 millones viven con diabetes tipo 1, por lo que se presume que para 2030 hará 578 millones de personas adultas con diabetes y para 2045 habrá 700 millones. Las prevalencias informadas por la FID en Latinoamérica, dos de los diez países con mayor número de casos se encuentran en Brasil y México. Doce países latinoamericanos tienen una prevalencia mayor al valor promedio mundial (8.3\%) (3).

En Sudamérica no se es ajena a esta realidad; en la región se estima una prevalencia de diabetes de $8 \%$ y se espera un aumento a 9,8\% para el año 2035. La prevalencia es menor en la población rural que en la urbana y las diferencias se 
atribuyen a los cambios en el estilo de vida (4).

En Ecuador según la Organización Panamericana de la Salud, la prevalencia de la diabetes es cada vez más elevada por lo que afecta a la población. De acuerdo a la encuesta ENSANUT, en las edades de 10 a 59 años la prevalecía de la diabetes es del $1.7 \%$, la misma que va subiendo desde los 30 años y a los 50, uno de cada diez ecuatorianos ya tiene diabetes. La población más afectada está entre las edades de 45 y 64 años. Es 15 veces mayor el riesgo de mutilaciones en pacientes diabéticos que en un paciente que no tiene esta enfermedad (4).

Salinero, y sus colaboradores en un estudio realizado el 2010 en Madrid con una muestra constituida por 261 pacientes de los cuales el 65,9\% eran mujeres. En la puntuación del cuestionario FINDRISC el $19,5 \%$ de los pacientes obtuvo un resultado mayor a 15 puntos (riesgo alto). Además, las mujeres presentaron un resultado del FINDRISC > 15 puntos en el $22,7 \%$ de los casos frente a un $13,5 \%$ de los hombres (5).

Brito, en un ensayo hecho en el año 2014 en una comunidad rural en Venezuela con una muestra constituida por 41 pacientes de los cuales el $61 \%$ eran mujeres; encontró que el $21,95 \%$ de los encuestados estaban en alto riesgo de desarrollar diabetes mellitus tipo 2, siendo las mujeres las más afectadas significativamente (6). Resultados que contrastan con lo encontrado por Paredes, et al. en el 2014 en una muestra de 404 individuos venezolanos de los cuales 304 eran mujeres, donde el $10,89 \%$ presentó riesgo alto de desarrollar diabetes (2).

La literatura sobre investigaciones para determinar el riesgo de desarrollar diabetes mellitus tipo 2 es muy escasa, por lo que no se pudo encontrar información a nivel Nacional y regional. Debido a la alta prevalencia de la diabetes mellitus tipo 2, según un estudio es la principal causa de morbilidad e incapacidad (7).

En ese escenario, la referida enfermedad crónica es considerada uno de los principales problemas de salud pública debido a la alta morbimortalidad proveniente de sus complicaciones crónicas, ocasionando gastos elevados de los servicios de salud para control y tratamiento de estos problemas, además de la reducción del personal trabajador $\mathrm{y}$ del impacto biopsicosocial en las personas afectada (8).

En resumen, según estudios se determina que los factores de riesgo genéticos, ambientales y metabólicos están relacionados y ayudan al desarrollo de la diabetes mellitus de tipo 2. Los antecedentes familiares de diabetes mellitus, la edad, la obesidad y la inactividad física identifican a los individuos con mayor riesgo. Las poblaciones minoritarias también corren un mayor riesgo, no sólo por los antecedentes familiares y la genética, sino también por la adaptación a los malos hábitos dietéticos y de ejercicio. Las mujeres con antecedentes de diabetes gestacional, así como sus hijos, tienen un mayor riesgo de desarrollar diabetes mellitus de tipo 2. La resistencia a la insulina aumenta el riesgo de que una persona desarrolle una intolerancia a la glucosa y una diabetes de tipo 2. Los individuos que tienen resistencia a la insulina comparten muchos de los mismos factores de riesgo que los que padecen diabetes de tipo 2. Entre ellos se encuentran la hiperinsulinemia, la dislipidemia aterogénica, la intolerancia a la glucosa, la hipertensión, el estado protrombótico, la hiperuricemia y el síndrome de ovario poliquístico. Las intervenciones para la prevención y el retraso de la diabetes mellitus de tipo 2 van encaminadas a transformar los factores de riesgo $y$ 
conciencia a promover el cribado, la detección precoz y el tratamiento en las poblaciones de alto riesgo con el objetivo de disminuir las complicaciones tanto microvasculares como macrovasculares (18).

Por lo expuesto anteriormente en la investigación se determinará el riesgo para desarrollar diabetes mellitus tipo II en las personas que residen en la ciudad de Cuenca, mediante el cuestionario FINDRISC. Los datos que se obtengan no ayudarán a la detección precoz del riesgo, la adopción de medidas preventivas y la administración temprana de tratamiento farmacológico, incluso durante el período subclínico de la enfermedad, se podría enlentecer el proceso patológico y el deterioro orgánico progresivo, con vistas a preservar la calidad de vida en la adultez.

\section{MATERIALES Y MÉTODOS}

$\mathrm{S}$ e realizó un estudio descriptivo, correlacional, prospectivo, cuantitativo y de corte transversal. La muestra se calculó mediante el muestreo aleatorio simple, la misma que estuvo constituida por 379 adultos entre hombres y mujeres de entre 18 y 65 años que residen en la ciudad de Cuenca, Ecuador que aceptaron participar voluntariamente y que firmaron el consentimiento informado. Y, se excluyeron en el estudio las personas con antecedentes patológicos personales de Diabetes Mellitus tipo 2.

Se utilizó la encuesta como técnica para la recolección de datos, en donde se incluyó los datos sociodemográficos de interés investigativo: edad, sexo, raza, estado civil, instrucción y ocupación. $Y$ el Test de FINDRISC que otorga una puntuación particular a los indicadores siguientes: edad, índice de masa corporal (IMC), perímetro de cintura, actividad física en el trabajo y/o en el tiempo libre, consumo de verduras o frutas, uso de medicamentos para la hipertensión arterial, antecedentes personales, episodios eventuales de hiperglucemia y antecedentes familiares, directos e indirectos, con diagnóstico de diabetes mellitus de tipos 1 o 2 .

El test de FINDRISC fue validado por Soriguer, Valdés, Tapia, Esteva, et al. que realizaron una investigación sobre la validación del FINDRISK en una población del sur de España, muestran que el FINDRISC es una herramienta útil para detectar sujetos con alto riesgo de diabetes en esta población.

Se realizó la investigación accediendo a la muestra de la ciudad de cuenca, perteneciente a la provincia del Azuay. Primeramente, se realizó visitas domiciliarias, donde se informó el tema y los objetivos de la investigación, siendo partícipes las personas que de forma voluntaria aceptaron participar y que firmaron el consentimiento informado. Se aplicó el test, que consta en recolectar las variables sociodemográficas, medidas antopométricas y suministrar el cuestionario de FINDRISC. Los datos de cada sujeto, poseen un código identificador de número de sujeto, en ningún caso se registró nombres, apellidos, número de cédula, correo electrónico, por lo tanto, los sujetos no pueden ser identificados.

Posteriormente se logró la recolección de los datos, los mismos que serán analizados y se podrá valorar por la tabla de calificación el riesgo de desarrollar Diabetes tipo 2 en un plazo de 10 años.

Se realizó un análisis descriptivo mediante porcentajes, frecuencias, medidas de tendencia central, posteriormente se realizó una prueba de normalidad mediante Shapiro Wilk (W) y la prueba de Levene, se 
pretendieron supuestos paramétricos. Por lo que se utilizó para la correlación el coeficiente de correlación de Pearson. Posteriormente, se efectuó un análisis de diferencia de medias mediante la prueba $\mathrm{T}$ de Student para muestras independientes. Para los análisis estadísticos antes mencionados se utilizaron los programas InfoStat y SPSS 26.

\section{RESULTADOS}

$\mathrm{E}$ n el estudio participaron un total de 379 adultos entre hombres y mujeres de entre 18 y 65 años que residen en la ciudad de Cuenca, Ecuador que cumplieron con los criterios determinados de inclusión y exclusión.

Tabla 1. Descripción de las características sociodemográficas de la población de estudio.

\begin{tabular}{llcc}
\hline Características & & f & \% \\
\hline Edad & $<45$ años & 264 & $69,7 \%$ \\
& entre 45 y 54 años & 72 & $19,0 \%$ \\
& Entre 55 y 64 años & 42 & $11,1 \%$ \\
Ocupación & $>64$ años & 1 & $0,3 \%$ \\
& Profesional & 66 & $17,4 \%$ \\
& Obrero & 13 & $3,4 \%$ \\
\multirow{3}{*}{ Sexo } & Campesino & 18 & $4,7 \%$ \\
& Otro & 282 & $74,4 \%$ \\
Etnia & Masculino & 133 & $35,1 \%$ \\
& Femenino & 246 & $64,9 \%$ \\
& Afro ecuatoriana & 0 & $0,0 \%$ \\
& Mestiza & 374 & $98,7 \%$ \\
Estado civil & Mulato & 0 & $0,0 \%$ \\
& Indígena & 4 & $1,1 \%$ \\
& Blanca & 1 & $0,3 \%$ \\
& Soltero & 180 & $47,5 \%$ \\
& unión libre & 36 & $9,5 \%$ \\
& Casado & 144 & $38,0 \%$ \\
& Viudo & 6 & $1,6 \%$ \\
& Divorciado & 13 & $3,4 \%$ \\
& Primaria & 102 & $26,9 \%$ \\
& Secundaria & 171 & $45,1 \%$ \\
& Superior & 106 & $28,0 \%$ \\
& Técnico & 0 & $0,0 \%$ \\
& Ninguno & 0 & $0,0 \%$ \\
& & &
\end{tabular}

Al analizar las características sociodemográficas se pudo determinar que de los 379 participantes el $69,7 \%$ tenían menos de 45 años, el $17,4 \%$ posee una profesión, el $64,9 \%$ es de sexo femenino, prevaleciendo la etnia mestiza con el $98,7 \%$, siendo el $47,5 \%$ el estado civil soltero predominante, el $45,1 \%$ tiene nivel de educación secundaria (Tabla 1). 
Tabla 2. Descripción de las características antropométricas.

\begin{tabular}{llcc}
\hline \multicolumn{1}{c}{ Características } & Media & Desv. \\
\hline Peso $(\mathrm{kg})$ & & 65,46 & 10,222 \\
Talla (m) & & 1,58 &, 076 \\
& & $\mathrm{f}$ & $\%$ \\
Perímetro cintura (cm) & 85 & $22,4 \%$ \\
& $<94 \mathrm{H},<80 \mathrm{M}$ & 114 & $30,1 \%$ \\
& $\mathrm{H}: 94-102 ; \mathrm{M}: 80-88$ & 180 & $47,5 \%$ \\
IMC & $\mathrm{H}>102 ; \mathrm{M}>88$ & 156 & $41,2 \%$ \\
& $<25 \mathrm{~kg} / \mathrm{m}^{2}$ & 180 & $47,5 \%$ \\
& $25-30 \mathrm{~kg} / \mathrm{m}^{2}$ & 43 & $11,3 \%$ \\
\hline
\end{tabular}

En relación a las características antropométricas, la población de estudio presentaba un peso corporal medio de $65,46 \mathrm{~kg}$, una talla media de $1,58 \mathrm{~m}$, el IMC más prevalente fue en el rango de 25 - 30 $\mathrm{kg} / \mathrm{m} 2$ con el 47,5\%, una mayor PC $\mathrm{H}>102 \mathrm{~cm} ; \mathrm{M}>88 \mathrm{~cm}$ el $47,5 \%$ (Tabla 2).

Tabla 3. Descripción de las características según estilos de vida.

\begin{tabular}{llcc}
\hline Estilo de vida & & f & \% \\
\hline Actividad física diaria al menos 30 minutos & Si & 109 & $28,8 \%$ \\
& No & 270 & $71,2 \%$ \\
Consume frutas /verduras diarias & Todos los días & 176 & $46,4 \%$ \\
& No todos los días & 203 & $53,6 \%$ \\
\hline
\end{tabular}

Respecto a los estilos de vida, estos reportaron menor actividad física diaria. En cuanto al consumo de alimentos, expusieron un menor consumo de frutas y verduras diarias (Tabla 3 ).
Se destaca que los participantes en porcentajes menores presentaron antecedentes familiares de DMT1 y 2 (Tabla 4).

Tabla 4. Descripción de las características según estilos de vida.

\begin{tabular}{llrr}
\hline Antecedentes & & f & \multicolumn{1}{c}{$\%$} \\
\hline HTA & No & 347 & $91,6 \%$ \\
& Si & 32 & $8,4 \%$ \\
Niveles altos de glucosa en sangre & No & 322 & $85,0 \%$ \\
& Si & 57 & $15,0 \%$ \\
Antecedentes familiares de DM1 y DM2 & No & 239 & $63,1 \%$ \\
& SI & 140 & $37,0 \%$ \\
\hline
\end{tabular}


El riesgo de desarrollar la DM2 en los próximos 10 años fue clasificado según la escala de FINDRISC en diferentes categorías: riesgo bajo, ligeramente aumentado, moderado, y alto.

El test de FINDRISC incluye: edad, IMC, circunferencia de la cintura, actividad física, consumo de verduras, frutas, hipertensión arterial, glucosa alta previa y antecedentes familiares de DM".
En lo relativo a la puntuación que se obtuvo según las respuestas de los participantes en el test de FINDRISC que evalúa el riesgo de desarrollar DM2 el $34,6 \%$ se encuentra en un riego ligeramente aumentado. En cuanto al riesgo bajo el $33,8 \%$. Observando que existe un porcentaje del $31,6 \%$ que se ubica en un riesgo modero - alto (Tabla 5).

Tabla 5. Descripción de la valoración del test FINDRISC.

\begin{tabular}{llrr}
\hline \multicolumn{2}{c}{ Valoración del test FINDRISC } & & \\
\hline \multirow{2}{*}{ Rangos } & $<$ f Riesgo Bajo & 128 & \% \\
& 7 a 11 Riesgo ligeramente aumentado & 131 & 33,8 \\
& 12 -14 Riesgo moderado & 74 & 34,6 \\
15 - 20 Riesgo Alto & 46 & 19,5 \\
& Total & $\mathbf{3 7 9}$ & 12,1 \\
& & & $\mathbf{1 0 0 , 0}$ \\
\hline
\end{tabular}

Se realizó la prueba de correlación $\mathrm{R}$ de Pearson entre las variables y la puntuación del test de FINDRISC, los resultados obtenidos se presentan en la Tabla 6. Se observa que las variables sexo, antecedentes familiares de DM1 y 2 y la edad presentan una correlación significativa con el riesgo según la puntuación del test de FINDRISC.

Tabla 6. Correlación $\mathrm{R}$ de Pearson entre la puntuación del test FINDRISC, género, antecedentes familiares de diabetes mellitus tipo II y rango de edad.

\begin{tabular}{llccc}
\hline \multicolumn{1}{c}{ Correlaciones } & & & \\
\hline & & Sexo & $\begin{array}{c}\text { Antecedentes } \\
\text { familiares de } \\
\text { DM1 y 2 }\end{array}$ & Edad \\
Riesgo según la puntuación & Correlación de Pearson &, $178^{* *}$ &, $369^{* *}$ &, $427^{* *}$ \\
del test FINDRISC & Sig. (bilateral) &, 000 &, 000 &, 000 \\
& $\mathrm{~N}$ & 379 & 379 & 379 \\
**. La correlación es significativa en el nivel 0,01 (bilateral). & & \\
\hline
\end{tabular}




\section{Asociación entre la valoración del test FINDRISC, edad, el peso, la talla, el perímetro de cintura y el tiempo diario de actividad física}

Se realizó la prueba de correlación $\mathrm{R}$ de Pearson entre las variables y la puntuación del test de FINDRISC, los resultados obtenidos se presentan en la Tabla 7. Se observa que las variables edad, el peso, la talla, el perímetro de cintura y el tiempo diario de actividad física. Presentan una correlación significativa con el riesgo según la puntuación del test de FINDRISC.

\begin{tabular}{lccccc}
\hline \multicolumn{5}{c}{ Correlaciones } \\
& edad & $\begin{array}{c}\text { peso } \\
\mathbf{( k g )}\end{array}$ & $\begin{array}{c}\text { talla } \\
\mathbf{( m )}\end{array}$ & PC (cm) & AF diaria \\
$\begin{array}{l}\text { Riesgo según la } \\
\text { puntuación del }\end{array}$ &, $427^{* *}$ &, $422^{* *}$ &,$- 275^{* *}$ &, $526^{* *}$ &, $394^{* *}$ \\
test FINDRISC &, $\mathbf{0 0 0}$ &, $\mathbf{0 0 0}$ &, $\mathbf{0 0 0}$ &, $\mathbf{0 0 0}$ &, $\mathbf{0 0 0}$ \\
& & & & & \\
$\begin{array}{l}\text { Correlación de Pearson; con negrita el valor de } p \\
\text { **. la correlación es significativa en el nivel } 0,01\end{array}$ & & & & \\
\hline
\end{tabular}

\section{Discusión}

Los resultados obtenidos en esta investigación la población, muestra una alta frecuencia de sobrepeso, obesidad abdominal, inactividad física, hábitos alimenticios inadecuados, siendo estos los factores de riesgo manifestados en esta población, los mismos que no se presentan como hechos aislados, sino que, por el contrario, que están relacionados con una marcada tendencia lo que a futuro podría desarrollar enfermedades como la DM2. Resultados similares fueron explicados en el estudio realizado por Cámara (9) demostrando que los factores de riesgo para desarrollar DM2 son el sedentarismo, exceso de peso, obesidad, glucosa en ayunas alterada, HTA, encontrando relación estadísticamente significativa entre variables. De la misma forma explica Regla (10) que existen factores de riesgo para desarrollar DM2: sobrepeso y obesidad, HTA, dislipidemia, sedentarismo y dieta no saludable.

Las posibilidades de desarrollar DM 2 obedecen de un conjunto de factores de riesgo, como la genética y el estilo de vida. Existiendo factores de riesgo no modificables como los antecedentes familiares, la edad o la etnia, sin embargo, si existen factores de riesgo que se pueden modificar relacionados con el estilo de vida, el tipo de alimentación, la actividad física y el peso para disminuir el riesgo de desarrollar DM2 (11).

En este estudio no se determinó casos de DM2, sin embargo, se determinó que el 15\% de los participantes tuvieron glicemia alterada en ayunas, lo cual los categoriza como casos de Prediabetes según la ADA. Tomando en cuenta que prediabetes concentra los casos de hiperglicemia que, aunque estén por encima de los rangos normales, no alcanzan el valor para considerarse DM2 (12). Esta condición no sólo implica un mayor riesgo de desarrollo de DM2, sino que también se ha demostrado que existe una asociación con otras patologías como enfermedades cardiovasculares y daño a nivel renal. Sim embargo se ha evidenciado que las alteraciones en glucemias son predictores 
de progresión a DM2. A este argumento Llorente (13) en su estudio que el riesgo de desarrollar diabetes mellitus tipo 2 es mayor en los pacientes con antecedentes familiares de la enfermedad, obesidad, hipertensión y portadores del síndrome metabólico.

Los resultados apoyan las directrices actuales sobre el peso que aconsejan a los adultos mantener su peso actual o, si tienen sobrepeso, perderlo, porque tanto el sobrepeso como la obesidad, así como el aumento de peso, se asocian con el desarrollo de la diabetes de tipo 2 (14).

\section{CONCLUSIONES}

$\mathrm{S}$ egún el objetivo planteado de la investigación se determinó el que el riesgo para desarrollar diabetes mellitus tipo II en las personas que residen en la ciudad de Cuenca es de prevalencia ligeramente aumentado, sin embargo, este riesgo tiende a incrementarse con la preexistencia de factores modificables y no modificables.

En la población estudiada, se determina que según la valoración de medidas antropométricas existen porcentajes altos de sobrepeso y obesidad, siendo uno de los factores principales de riesgo para desarrollar diabetes mellitus tipo II.

Los malos hábitos alimenticios, los antecedentes personales de hiperglucemias, antecedes familiares de diabetes y la HTA son factores predisponentes para desarrollar MD2.

Se establece que el porcentaje de riesgo para desarrollar diabetes mellitus tipo II está asociado a la edad, el peso, la talla, el perímetro de cintura y el tiempo diario de actividad física.
Los resultados de este estudio recomiendan invertir en investigación y sistemas de salud en la Ciudad de Cuenca para frenar los factores relacionados con la diabetes. Hacer hincapié en la mejora y el avance de los programas de educación en los medios de comunicación para sensibilizar al público sobre las complicaciones de la diabetes de tipo 2. Incentivar a la población para que realice ejercicio con regularidad para reducir la masa corporal, siendo este un factor de riesgo continuo para desarrollar diabetes.

Aunque los antecedentes familiares aumentan el riesgo de contraer diabetes de tipo 2, se ha visto que el ajuste de los factores ambientales, como el estilo de vida, entre otros, lo altera. Por lo tanto, se debe realizar una reeducación sobre la alimentación y la importancia de realizar ejercicio con regularidad.

\section{REFERENCIAS BIBLIOGRÁFICAS}

1. Conesa A, González T. Aspectos más recientes en relación con la diabetes mellitus tipo MODY. Revista Cubana de Endocrinología. 2013 [citado: 10 de diciembre de 2020]; 23(3):186-94. Disponible en: http://scielo.sld.cu/scielo.php?script=sci_a rttext\&pid=S1561-29532012000200008

2. ALAD. Guías ALAD sobre diagnóstico, control y tratamiento de la Diabetes Mellitus Tipo 2 con Medicina Basada en Evidencia. Revista de la Asociación Latinoamericana de Diabetes. 2013[citado: 11 de diciembre de 2020]; 17-128. Disponible en: https://revistaalad.com/guias/5600AX19 1_guias_alad_2019.pdf

3. Agudelo-Botero M, Dávila-Cervantes C. Carga de la mortalidad por diabetes mellitus en América Latina 2000-2011: los casos de Argentina, Chile, Colombia y 
México. Revista Gac Sanit. 2015[citado: 11 de diciembre de 2020]; 29(3):172-177. Disponible en: http://scielo.isciii.es/scielo.php?script=sci _arttext\&pid=S0213-91112015000300003

4. Iglesias R, Barutell L, Artola S, Serrano R. Resumen de las recomendaciones de la American Diabetes Association (ADA) 2014 para la práctica clínica en el manejo de la diabetes mellitus. Diabetes Práctica. 2014[citado: 12 de diciembre de 2020]; 5(2):1-24. Disponible en: http://www.bvs.hn/Honduras/UICFCM/Di abetes/ADA.2014.esp.pdf

5. Swoboda P, McDiarmid A, Erhayiem B, Ripley D, Dobson L, Garg P, et al. 029 Diabetes, microalbuminuria y cardiopatía subclínica: identificación y seguimiento de personas con riesgo de insuficiencia cardíaca. Revista Heart. 2017[citado: 12 de diciembre de 2020]; 103(1): A25-A25. Disponible en: http://heart.bmj.com/lookup/doi/10.113 6/heartjnl-2017-311399.29

6. Brito-Núñez N, Brito-Núñez J, RuizRendón C. Riesgo de diabetes en una comunidad rural del municipio Sotillo.Estado Monagas.Venezuela. Revista Venezolana de Endocrinología y Metabolismo. 2014[citado: 20 de diciembre de 2020]; 12(3): 167-176. Disponible en: http://ve.scielo.org/scielo.php?script=sci arttext\&pid=S1690-31102014000300004

7. Menke A, Casagrande S, Geiss L, Cowie CC. Prevalencia y tendencias de la diabetes entre adultos en los Estados Unidos, 19882012. Revista Jama 2015[citado: 22 de diciembre de 2020]; 314(10):1021- 1029. Disponible en: http://jama.jamanetwork.com/article.aspx ?doi=10.1001/jama.2015.10029

8. Oliveira P, Costa M, Ferreira J, Lima C. Autocuidado en Diabetes Mellitus: estudio bibliométrico. Enferm Glob. 2017 [citado: 22 de diciembre de 2020]; 1(45):634-652. Disponible en: https://revistas.um.es/eglobal/article/vie $\mathrm{w} / 249911$
9. Lima A, Araújo M, Freitas R, Zanetti M, Almeida P, Damasceno M. Factores de riesgo para Diabetes Mellitus Tipo 2 en universitarios: asociación con variables sociodemográficas. Rev. Latino-Am. Enfermagem. 2014 [citado 22 Mar 21]; 22(3):484-490. Disponible en: http://www.scielo.br/scielo.php?script=sc i_arttext\&pid=S010411692014000300484\&lng=en. http://dx.doi.org/10.1590/01041169.3053.2441

10. Regla I, Molena $C$, Tasca $R$, Marcon S, Cuman R. Factores de riesgo en pacientes con diabetes mellitus tipo 2. Rev. LatinoAm. Enfermagem. 2008 [citado 22 Mar 21]; 16(2): 238-244. Disponible en: http://www.scielo.br/scielo.php?script=sc i_arttext\&pid=S0104$11692008000200011 \& \operatorname{lng}=\mathrm{en}$. http://dx.doi.org/10.1590/S010411692008000200011

11. Bohórquez $C$, Barreto $M$, Muvdi $Y$, Rodríguez A, Badillo A, Martínez de la Rosa Walter Ángel et al. Factores modificables y riesgo de diabetes mellitus tipo 2 en adultos jóvenes: un estudio transversal. Cienc. enferm. 2020 [citado 22 Mar 21]; 26: $14 . \quad$ Disponible en: https://scielo.conicyt.cl/scielo.php?script= sci_arttext\&pid=S0717-

95532020000100210\&lng=es. Epub 13Oct-2020.

http://dx.doi.org/10.29393/ce267 fmcb70007

12. Valdés $W$, Almirall $A$, Gutiérrez $M$. Factores de riesgo de diabetes mellitus tipo 2 en adolescentes. Medisur. 2019 Jun [citado 22 Mar 21]; 17(3): 356-364. Disponible en: http://scielo.sld.cu/scielo.php?script=sci_a rttext\&pid=S1727-

897X2019000300356\&lng=es. Epub 02Sep-2019

13. Llorente $Y$, Miguel $P$, Rivas D, Borrego Y. Factores de riesgo asociados con la aparición de diabetes mellitus tipo 2 en personas adultas. Rev Cubana Endocrinol. 2016 [citado 22 Mar 21]; 27(2). Disponible 
http://scielo.sld.cu/scielo.php?script=sci_art text\&pid=S1561-29532016000200002 \&lng $=\mathrm{es}$

14. Hernández E, Batlle $M$, Martínez B, et al. Cambios alimentarios y de estilo de vida como estrategia en la prevención del síndrome metabólico y la diabetes mellitus tipo 2: hitos y perspectivas. Anales Sis San Navarra [Internet]. 2016 [citado 22 Mar 21]; 39(2): 269-289. Disponible en: http://scielo.isciii.es/scielo.php?script=sci_a rttext\&pid=S1137-66272016000200009\& lng=es.
Conflicto de intereses. No existen conflictos personales, profesionales o de otro tipo.

Fuente de Financiamiento. Los autores no declaran.

Agradecimiento. A la Coordinadora y Docentes de la Maestría en Gestión del Cuidado de la Universidad Católica de Cuenca y al Laboratorio de Psicometría, Psicología Comparada y Etología del Centro de Investigación, Innovación y Transferencia de Tecnología (CIITT). 